\title{
EDITORIAL
}

\section{Memory of Professor Hamao Umezawa}

\author{
The Journal of Antibiotics (2018) 71, 2-3; doi:10.1038/ja.2017.137
}

$\mathrm{T}_{\mathrm{r}}^{\mathrm{n}}$ he discovery of antibiotics in the 20th century is among the most I remarkable achievements with the widest benefits to mankind. A wide variety of antibiotics are now available to treat previously fatal bacterial infections, revolutionizing medical care.

Dr Hamao Umezawa began research on new antibiotics in the 1940s, producing the first penicillin in Japan and later discovering kanamycin, the first broadly effective agent against streptomycinresistant Mycobacterium tuberculosis infection (Photo 1).

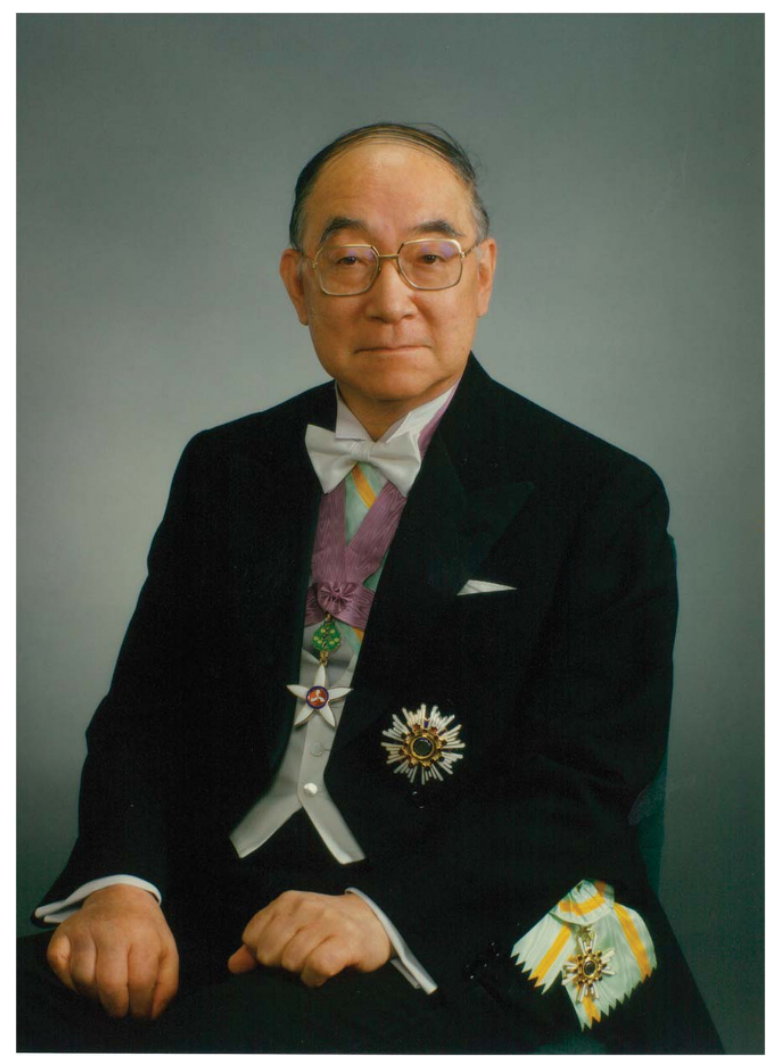

Professor Hamao Umezawa.
In addition, he pioneered the use of antibiotics as anticancer agents. From the latter half of the 1940s, he developed entirely new research fields for over 40 years while remaining a world leader in antibiotics research. In addition to kanamycin, he discovered more than 70 antimicrobial antibiotics including arbekacin and josamycin, more than 40 anticancer antibiotics including bleomycin and aclarubicin, more than 50 enzyme inhibitors including leupeptin, pepstatin and antipain, the antibiotic and antifungal agent kasugamycin (a control agent for the rice blast disease), and several immunomodulators including ubenimex.

Through these discoveries, he made substantial contributions not only to medicine but also to basic pharmacology, biochemistry and agriculture. Furthermore, Dr Umezawa developed a logical research pathway to obtain new effective compounds based on bacterial resistance mechanisms. In particular, while studying kanamycin resistance mechanism, he discovered inactivating mechanisms mediated by phosphotransferases and acetylases. Based on this result, he synthesized dibekacin, an antibiotic not affected by these enzymes. He also succeeded in developing arbekacin, which overcomes dibekacin resistance by adenylation.

Dr Umezawa believed that research on therapeutic drugs is 'research that decides that there should be an answer to a question that does not understand whether there is an answer.' He developed medicines that responded to current demands and provided a research path for others to follow. Dr Umezawa established the Institute of Microbiological Chemistry in 1962, and in the decades since, he and many colleagues have immensely contributed to the advancement of medicine and life sciences, particularly in the field of antibiotics research through its organizational strength. We honor Dr Umezawa's legacy by continuing to develop new antimicrobial agents and related compounds for disease without effective treatments, such as drug-resistant tuberculosis, multidrug-resistant Gram-negative bacteria, and viral and protozoal infections. Through these efforts, our institute continues to promote scientific achievement and human welfare (Photo 2). 


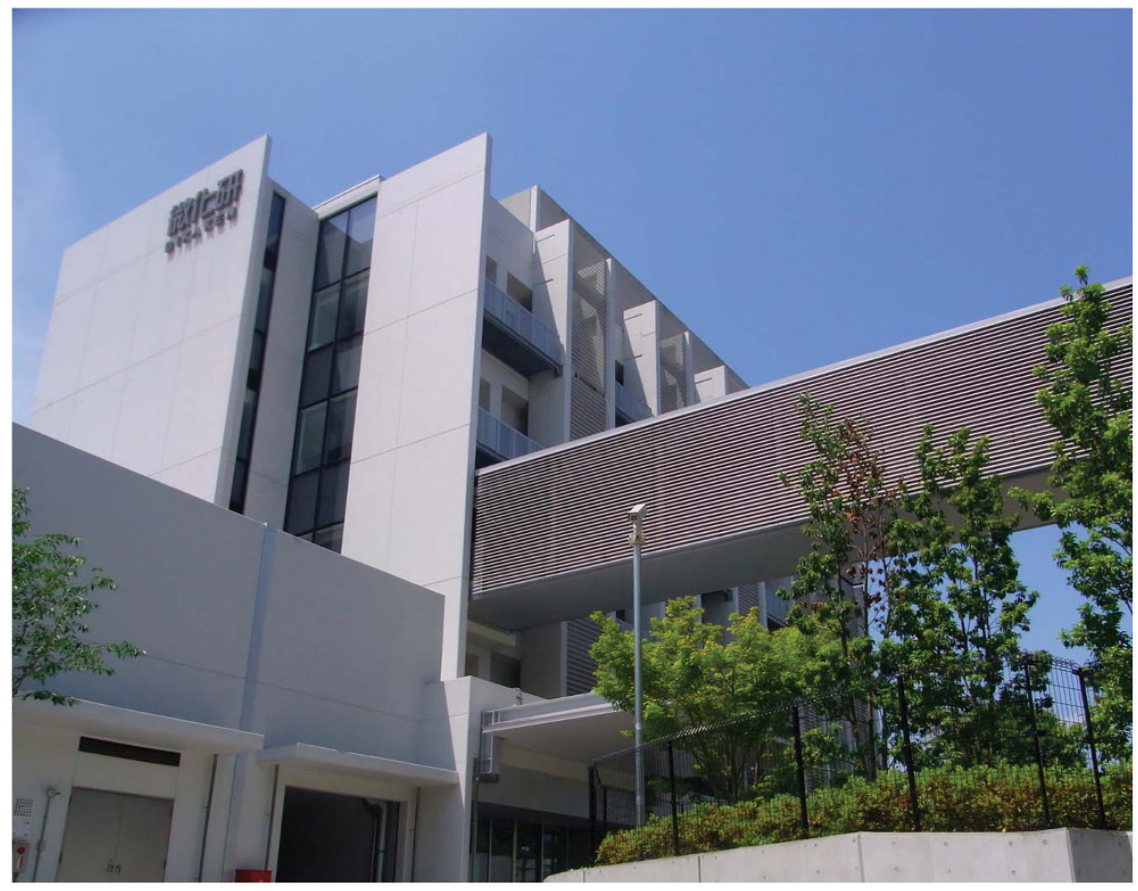

The Institute of Microbiological Chemistry, a new research building was constructed in May 2015

\section{CONFLICT OF INTEREST}

The authors declare no conflict of interest.
Masakatsu Shibasaki ${ }^{1}$ and Katsuhisa Yamazaki ${ }^{2}$ ${ }^{1}$ Institute of Microbial Chemistry, Tokyo, Japan and ${ }^{2}$ Section of Intellectual Property and Public Relations, Institute of Microbial Chemistry, Tokyo, Japan E-mail:yamazakik@bikaken.or.jp 